\title{
Targeting survival pathways to create infarct-spanning bridges of human embryonic stem cell-derived cardiomyocytes
}

\author{
Jun Luo, MD, PhD, ${ }^{a}$ Matthew S. Weaver, $\mathrm{PhD},{ }^{\mathrm{a}}$ James E. Dennis, $\mathrm{PhD},{ }^{a}$ Elizabeth Whalen, $\mathrm{PhD},{ }^{\mathrm{a}}$ \\ Michael A. Laflamme, MD, PhD, ${ }^{b}$ and Margaret D. Allen, MD ${ }^{a, b}$
}

\begin{abstract}
Objective: Generating myocyte grafts that bridge across infarcts could maximize their functional impact and best utilize small numbers of stem cells. To date, however, graft survival within acute infarcts has not been feasible. To enhance intrainfarct graft viability, human embryonic stem cell-derived cardiomyocytes (hESC$\mathrm{CMs}$ ) were pretreated before implantation with cobalt protoporphyrin (CoPP), a pharmacologic inducer of cytoprotective heme oxygenase-1.
\end{abstract}

\begin{abstract}
Methods: After preculturing with CoPP (vs phosphate-buffered saline), hESC-CMs were injected intramyocardially into acutely infarcted rat hearts, using directed injections to span the infarct. A further group received CoPP-pretreated hESC-CMs plus 4 weekly doses of systemic CoPP to prolong exposure to cytoprotectants. Two control groups with infarcts received vehicle-only intramyocardial injections or weekly systemic CoPP without cell therapy. Postinfarct ventricular function was gauged by echocardiography and graft size quantified at 8 weeks by histomorphometry.
\end{abstract}

Results: CoPP-preconditioned hESC-CMs formed stable grafts deep within infarcted myocardium, while grafts without CoPP exposure survived mainly at the infarct periphery. Fractional shortening was improved at 4 and 8 weeks in all hearts receiving cell therapies $(P<.01$ vs vehicle-only injections). CoPP treatment of both graft $\mathrm{hESC}-\mathrm{CMs}$ and recipient animals resulted in the largest grafts, highest fractional shortening, preserved wall thickness, and reduced infarct dimensions.

Conclusions: Cellular therapy delivered acutely after infarction significantly improved postinfarct ventricular function at 1 and 2 months. CoPP pretreatment of cells resulted in stable hESC-CM grafts within infarcted myocardium. This design enables construction of directionally oriented, infarct-spanning bands of new cardiomyocytes that might further improve functional restoration as engrafted myocytes proliferate and mature. (J Thorac Cardiovasc Surg 2014;148:3180-8)

See related commentary on pages 3188-93.

Video clip is available online.

Supplemental material is available online.

From the Benaroya Research Institute at Virginia Mason, ${ }^{\text {a }}$ and University of Washington School of Medicine, ${ }^{\mathrm{b}}$ Seattle, Wash.

This study was supported by the National Institutes of Health (grant R01 HL064387 to M.D.A. and M.A.L. and grant R01 HL086709 to M.D.A).

Disclosures: James E. Dennis reports consulting fees from Biotime Inc. Michael A. Laflamme reports equity ownership in BEAT Biotherapeutics. All other authors have nothing to disclose with regard to commercial support.

Drs J.L. and M.S.W. contributed equally to this study.

Received for publication April 23, 2014; revisions received May 29, 2014; accepted for publication June 18, 2014; available ahead of print Sept 16, 2014.

Address for reprints: Margaret D. Allen, MD, Benaroya Research Institute at Virginia Mason, 1201 Ninth Ave, Seattle, WA 98101 (E-mail: mallen@benaroyaresearch. org).

$0022-5223 / \$ 36.00$

Copyright $($ c 2014 by The American Association for Thoracic Surgery

http://dx.doi.org/10.1016/j.jtcvs.2014.06.087
In the normal heart, ventricular cardiomyocytes are organized into macroscopic helical myocardial bands that produce the twisting systolic movement, or torsional deformation, of the left ventricle during contraction. ${ }^{1-4}$ Myocardial infarction (MI) destroys these oriented myocardial fibers, damaging the structural architecture of the ventricle. Current intramyocardial cell delivery techniques produce disconnected clusters of randomly oriented grafts. We examined whether, instead, it would be possible to generate band-like cardiomyocyte grafts to span the infarct area. Such graft bridges could reconnect the inherent ventricular myocardial bands still present at the infarct borders, thus maximizing the functional contribution of the new cells.

However, a major limitation for cell-based therapies is that only $10 \%$ to $20 \%$ of injected cells survive implantation into ischemic infarcts, with most cell loss occurring within the first week. ${ }^{5-8}$ Graft cell survival within an acute infarct has not previously been a realistic expectation $^{5,6,9,10}$ and, consequently, cell injections are commonly delivered into peri-infarct areas. ${ }^{7,11,12}$ The goal of the present study was to determine whether pharmacologic induction of survival factors could improve graft survival within the infarct, which, in turn, would allow construction of infarct-spanning cellular bridges. 


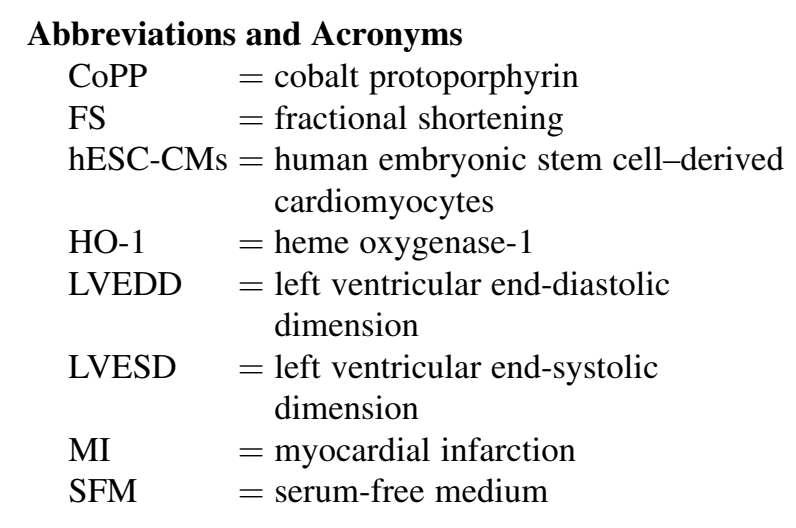

Our group has previously shown that pretreating cardiomyocyte grafts with cobalt protoporphyrin (CoPP) markedly improves their survival after implantation into an ischemic environment. ${ }^{12,13}$ CoPP activates the transcription factor, nrf2, which initiates transcription of cytoprotective heme oxygenase-1 (HO-1) and additional antioxidative factors. ${ }^{14,15}$ Preemptive HO-1 induction by gene therapy has been shown to reduce infarct size, myocyte apoptosis, and remodeling in hearts subjected to myocardial infarction. ${ }^{16-19}$ The downstream end products of HO-1, bilirubin and carbon monoxide, are antiapoptotic, antioxidative, proangiogenic, and antifibrotic ${ }^{20,21}$-all desirable attributes for cell therapy in ischemic beds. In these experiments, we applied ex vivo CoPP treatment to human embryonic stem cellderived cardiomyocytes ( $\mathrm{hESC}-\mathrm{CMs}$ ), a cell population with potential use in clinical cell therapy. Our laboratory has found that exposing hESC-CMs to a single dose of CoPP produces sustained HO-1 upregulation for at least 4 days. ${ }^{12}$ Thus, ex vivo CoPP pretreatment of cells prior to implantation would, potentially, provide grafted cells with a survival advantage during the critical first few days following implantation. Besides CoPP pretreatment of graft cells, a month-long course of systemic CoPP was administered to some recipient groups as a means to extend the pharmacologic effects during early infarct maturation.

\section{METHODS}

\section{Preparation and Characterization of hESC-CMs}

HESC-CMs were generated from the $\mathrm{H} 7$ human embryonic stem cell line $^{22}$ by serial application of activin A (R\&D Systems, Minneapolis, Minn) and BMP4 (R\&D Systems) ${ }^{10}$ omitting the "pro-survival" cocktail and Percoll gradient centrifugation. ${ }^{12}$ Spontaneous contraction was observed after additional culture in RPMI-B27 serum-free medium (SFM) (Invitrogen, Carlsbad, Calif). For HO-1 induction, the cell culture medium was supplemented with $25 \mu \mathrm{M}$ CoPP (Frontier Scientific Inc, Logan, Utah) in phosphate-buffered saline (PBS, Invitrogen). Control hESC-CMs were cultured in media supplemented with PBS alone. Cells were then enzymatically dispersed and cryopreserved until implantation.

To characterize cells just prior to implantation, aliquots from each thawed cell batch (Video 1) were plated and fixed with methanol for immunocytochemical profiling. Nascent cardiomyocytes were identified with antibodies to cardiac troponin I (Abcam, San Francisco, Calif) and human Nkx2.5 (R\&D Systems), an early cardiac-specific transcription factor; mitotic cells with antibody to Ki67 (Abcam); and endothelial cells with antibody to human CD31 (Dako Inc, Carpinteria, Calif). Nuclei were counterstained with Hoechst 33342 dye (Invitrogen).

\section{Permanent MI Model}

Animal protocols were approved by the Institutional Animal Care and Use Committee and conducted in accordance with the Guide for the Care and Use of Laboratory Animals (National Research Council 2011). Rats were placed under isoflurane anesthesia and mechanically ventilated for MI surgery. Through a left thoracotomy, the left anterior descending coronary artery was permanently ligated without reperfusion. Five minutes after coronary ligation, microspheres, cells, or media were injected into the infarct, followed by chest closure and recovery from anesthesia.

\section{Microsphere Retention After Intramyocardial Injection}

Microspheres, equivalent in size to hESC-CMs, were injected into acute MIs to model immediate cell loss from leakage and washout after direct intramyocardial injection. In 5 Fischer 344 rats (Charles River Laboratories, Portage, Mich), $5 \times 10^{6}$ Hydro-Coated Yellow E-Z Trac Ultraspheres (15- $\mu \mathrm{m}$ diameter; Interactive Medical Technology, Irvine, Calif) suspended in $70 \mu \mathrm{L}$ RPMI were injected into the infarct centers; the heart, lung, and spleen were excised 15 minutes later. In heart specimens, yellow microspheres were imaged by dark field epi-illumination under fluorescence stereomicroscopy (Leica Microsystems, Wetzlar, Germany). Whole hearts, lungs, and spleens were placed into individual centrifuge tubes to which equal numbers of Pink E-Z Trac Ultraspheres $\left(5 \times 10^{6}\right.$ in $70 \mu \mathrm{L}$ RPMI) were added as the standard for $100 \%$ recovery. After microsphere extraction, the relative proportion of injected (yellow) to standard (pink) microspheres in each organ was quantified by flow cytometry.

\section{Implantation of hESC-CMs Into Infarcted Hearts}

For implantation, $1 \times 10^{7} \mathrm{hESC}-\mathrm{CMs}$, suspended in $100 \mu \mathrm{L}$ RPMI SFM, were loaded into a $0.3-\mathrm{mL}$ syringe with a 29 -gauge needle for intrainfarct injection into athymic male rats (NIH-Whn, 240-280 g; Taconic Farms, Cambridge City, Ind). To produce a band of cells across the infarct, the needle was inserted from each of the 2 infarct margins, converging at the infarct center, creating a track that spanned the infarct from 1 uninjured edge to the other (Video 2). Cells were delivered as the needle was withdrawn from the myocardium, ${ }^{23}$ rather than during needle insertion, to facilitate even distribution along the needle track.

\section{Treatment Groups}

Athymic rats, all with acute infarcts, were randomized into 5 treatment groups ( $\mathrm{n}=8 /$ group). Three cell treatment groups received intrainfarct injections of hESC-CMs: group 1, hESC-CMs pretreated ex vivo with PBS; group 2, hESC-CMs pretreated ex vivo with $25 \mu \mathrm{M}$ CoPP for 24 hours before implantation; and group 3, hESC-CMs pretreated with CoPP plus recipient rat treatment with a 4-week course of systemic CoPP $(5 \mathrm{mg} / \mathrm{kg} /$ dose intraperitoneally, delivered 24 hours before surgery, then once weekly). Two rats died following surgery ( 1 each from groups 1 and 2) and were replaced; all were included in the baseline data. Two control groups did not receive cell therapy. Of these, 1 control group (group 4) received intrainfarct injections of vehicle (SFM) alone, while the second control group (group 5) received the 4-week course of systemic CoPP but without cell therapy. This intraperitoneal dose of CoPP, ${ }^{24-26}$ given weekly, ${ }^{27}$ produces sustained HO-1 upregulation in heart tissue.

\section{Echocardiography}

Two-dimensional M-mode echocardiograms were performed under isoflurane anesthesia 24 hours before surgery (preinfarction baseline) and at 48 hours, 4 weeks, and 8 weeks after surgery (M-Turbo; SonoSite, Inc, 
Bothell, Wash). Percent fractional shortening (FS) was calculated from the left ventricular end-diastolic (LVEDD) and end-systolic (LVESD) dimensions as [(LVEDD - LVESD)/LVEDD] $\times 100$.

\section{Histologic Evaluation of hESC-CM Grafts}

At 8 weeks after surgery, hearts were excised, fixed in methyl Carnoy's solution, paraffin embedded, and sectioned at 1-mm intervals between the coronary ligation site and ventricular apex. Serial 5- $\mu$ m-thick sections within each macrosection were treated with hematoxylin and eosin and Masson's trichrome stains. Human cellular grafts were identified with antibodies to the $\beta$-myosin heavy chain isoform (ATCC, Manassas, Va), which is preferentially expressed in human, but not rat, myocardium ${ }^{6,10}$ and with human-specific antibody to lamin A/C (Millipore, Billerica, Mass). ${ }^{28}$ Gap junctions were labeled with antibodies to connexin 43 (Cell Signaling, Danvers, Mass). Nuclei were counterstained with Hoechst dye.

\section{Morphometric Assessments}

Morphometry was performed on 4 transverse macrosections per heart, encompassing the entire infarct. Planimetry was performed using ImageJ (National Institutes of Health, Bethesda, Md), utilizing Masson's trichrome staining, which delineates fibrillar collagen, to define the infarct. Infarct size was quantified as the mean percentage of left ventricular circumference occupied by the trichrome blue-positive infarct zone ${ }^{29-31}$ over all sections for each heart. Infarct wall thickness was calculated as the mean of 3 measurements taken across the infarct, divided by the mean of 2 measurements of the noninfarcted adjacent ventricular wall. ${ }^{32}$

Graft size was measured as the cumulative sum of $\beta$-myosin heavy chain-positive areas on these same standardized sections. The percentage of grafted area within the infarct was quantified as the sum of grafted areas surrounded by collagen-rich infarct tissue on each section divided by the total graft area. The extent of infarct replacement by grafted cells was determined as the percentage of the total infarct area occupied by human lamin A/C-positive graft cells.

\section{Statistical Analysis}

Data are expressed as means \pm standard deviations. One-way analyses of variance with post hoc Bonferroni corrections were used to assess single outcome measures among treatment groups at the 8-week time point. Significance levels were adjusted to $P<.017$ ( 0.05 divided by 3 ) and $<.005$ ( 0.05 divided by 10) for comparisons among 3 and 5 treatment groups, respectively. Analysis of longitudinal echocardiographic data was performed in R statistical software (The R Project for Statistical Computing; available at: www.r-project.org) using the Laird-Ware mixed effects model. ${ }^{33}$ The model was fitted with indicator variables for the 5 treatment groups and 4 measurement points and 12 two-way interaction terms between these variables, and included the random effect of animal identification number to estimate the variability caused by individuals. Two-way analyses of variance were used to evaluate 4 outcome variables with Bonferroni multiple testing corrections, using $P<.0125$ ( 0.05 divided by 4$)$ to determine significance.

\section{RESULTS}

\section{Characterization of Injectates Prior to Implantation}

Cardiomyocytes (cardiac troponin I-positive) comprised $64 \% \pm 4 \%$ of cells across all aliquots (Figure E1, $A$ ). Twenty-seven percent $\pm 6 \%$ of cells stained positively for the cardiac-specific transcription factor, $\mathrm{Nkx} 2.5$, indicating some cardiomyocytes still in an early phase of differentiation; $15 \% \pm 8 \%$ of cells were Ki67-positive, denoting a mitotic population (Figure E1, $B$ and $C$ ). Human endothelial-like cells (hCD31-positive) were rare $(<0.1 \%$; data not shown). No difference in cell composition was seen between CoPP-pretreated and PBS-pretreated injectates.

\section{Injectate Retention Within the Heart After Intrainfarct Injection}

Recovery of cell-sized microspheres was used to estimate the extent of immediate cell loss after intrainfarct injection. Fifteen minutes after delivery, $68 \% \pm 16 \%$ of injected microspheres were retained within the heart, the majority situated along the needle injection track (Figure 1); 10\% $\pm 5 \%$ had dispersed to the lungs $(P<.0001$ vs heart specimens), and no signal was detected in the spleen $(0.001 \pm$ $0.001, P<.0001$ vs heart specimens). Immediate microsphere loss (and, hence, expected cell loss) through leakage and intramyocardial collateral flow thus totaled about $30 \%$ of the injectate, with the redistribution primarily into the pulmonary, not the systemic, circulation.

\section{Graft Histology at 8 Weeks Postimplant}

At 8 weeks after implantation, surviving hESC-CM grafts were found in all hearts receiving hESC-CM injections (Figure 2, $B$ - $G$ ), corroborated by $\beta$-myosin heavy chain immunostaining (Figure 2, $H$ ). Immunostaining with human-specific lamin $\mathrm{A} / \mathrm{C}$ further confirmed these grafts to be of human origin (Figure 2,I). By this point, gap junction proteins were well expressed within the grafts (Figure 2, $J$ ).

\section{Grafts of CoPP-Pretreated hESC-CMs Are Primarily Located Within the Infarct Center}

What most distinguished CoPP-pretreated hESC-CM grafts from PBS-pretreated controls was the extent to which cells engrafted within the infarct center (Figure 3, A). By 8 weeks postimplant, three quarters of the total graft area in CoPP-pretreated grafts was located deep within the infarct ( $76 \% \pm 9 \%$ for CoPP-pretreated grafts; $77 \% \pm 12 \%$ for CoPP-pretreated grafts plus systemic CoPP). In contrast, surviving PBS-pretreated hESC-CMs were found primarily at the infarct margins with just $38 \% \pm 11 \%$ of the graft area within the infarct center $(P<.0001$ vs each CoPP-treated group).

\section{Adding Systemic CoPP Increases Graft Size at 8 Weeks}

At 8 weeks, the rats receiving CoPP-pretreated hESC-CMs plus a 4-week course of systemic CoPP had larger cumulative graft areas $\left(0.19 \pm 0.02 \mathrm{~mm}^{2}\right)$, effectively double those in the 2 other cell therapy groups $(P<.0001$ vs each group; Figure $3, B)$. Without systemic CoPP, graft sizes in hearts receiving CoPP-pretreated hESC-CMs were similar to those receiving PBS-pretreated cells $\left(0.10 \pm 0.03 \mathrm{~mm}^{2}\right.$ vs $0.11 \pm 0.00 \mathrm{~mm}^{2}$, respectively; $P=.67)$. 

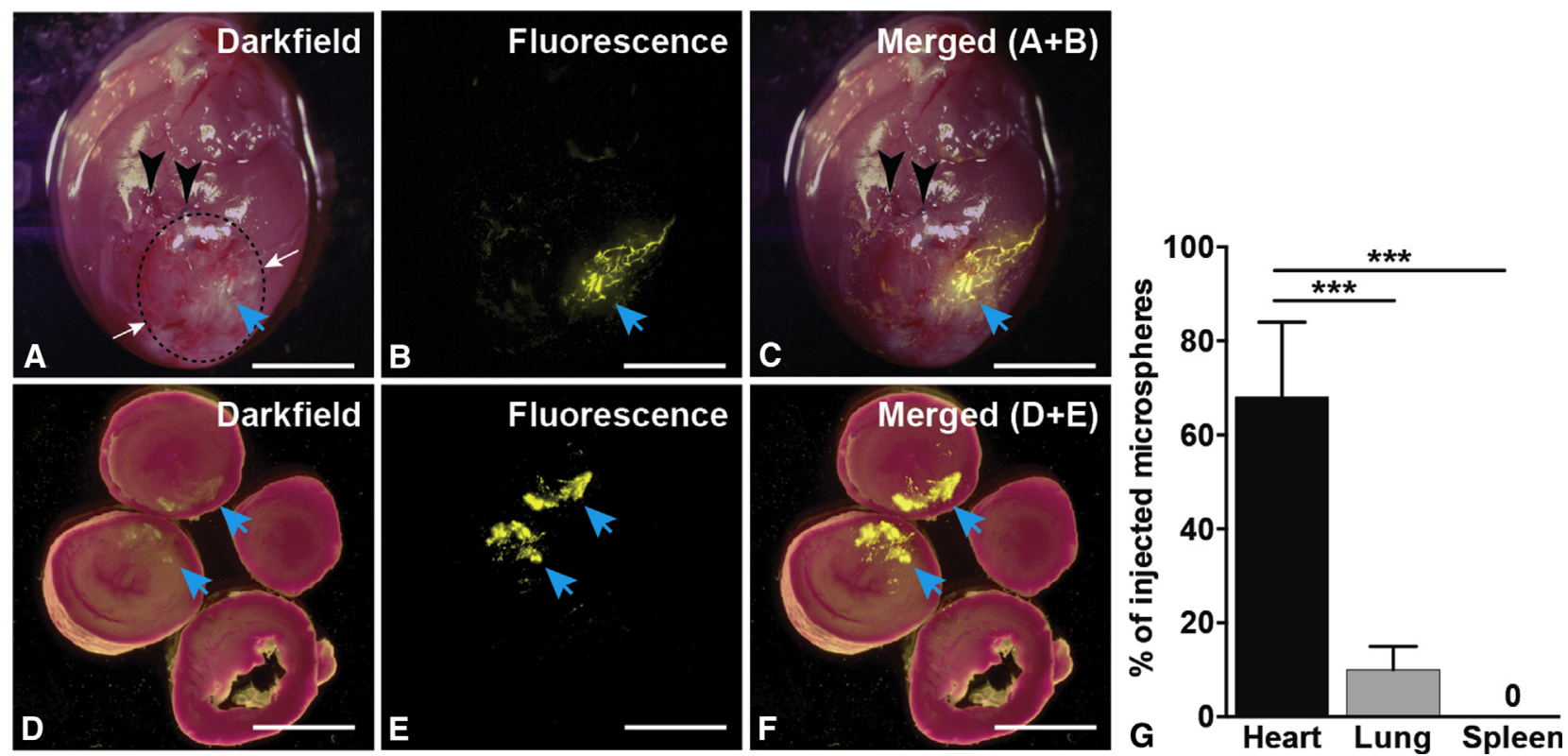

FIGURE 1. Microsphere biodistribution after intrainfarct injection. Representative example of an infarcted heart 15 minutes after intramyocardial injection of yellow fluorescent microspheres. A-C, Gross specimen showing the anterior left ventricle with infarct (circled). Black triangles mark ligation sites on the left anterior descending and diagonal coronary arteries. White arrows indicate the direction of transinfarct needle injection. $\mathrm{C}$, Blue arrows point to the majority of the microspheres, which remain within the infarct along the injection tract, effectively spanning the infarct. D-F, Consecutive cross-sections through the same heart illustrating the microspheres within the mid-level sections. A and D, Dark field epi-illumination images; B and E, fluorescence imaging to visualize microspheres; $\mathrm{C}$ and $\mathrm{F}$, merged images. Scale bar $=5 \mathrm{~mm}$. G, Relative microsphere retention in heart, lung, and spleen as quantified by flow cytometry $(\mathrm{n}=5)$. ***P $<.0001$ versus lung or spleen.

\section{CoPP Treatment Increases Infarct Replacement by Engrafted Cells}

Planimetry revealed that a larger percentage of the infarct area was composed of graft cells in hearts receiving CoPP-pretreated versus PBS-pretreated hESC-CMs $(8.2 \%$ $\pm 0.8 \%$ vs $6.1 \% \pm 1.0 \% ; P<.001$; Figure $3, C$ ). Infarct replacement by graft cells was further increased when CoPP pretreatment of the graft was combined with a 4-week course of systemic CoPP $(9.7 \% \pm 0.8 \%, P<.01$ vs CoPP-pretreated, and $P<.0001$ vs PBS-pretreated grafts; Figure 3,C).

\section{Infarct Size Is Reduced in Hearts Receiving CoPP- Pretreated hESC-CMs}

Histomorphometry at 8 weeks revealed that infarct sizes ranged from $29 \% \pm 3 \%$ to $37 \% \pm 3 \%$ of the ventricular circumference across all groups, confirming that all hearts had sustained substantial infarction. Infarct size was not altered by cell therapy alone, as the infarct sizes in the hearts with PBS-pretreated hESC-CMs $(35.2 \% \pm 2.5 \%)$ were not smaller than in hearts receiving vehicle-only (SFM) injections $(37.2 \% \pm 3.0 \%, P>.9999)$. Also, a transient, 4-week course of systemic CoPP, by itself, did not affect infarct size at 8 weeks $(37.1 \% \pm 3.0 \%, P>.9999$ vs vehicle-only controls). In contrast, however, infarct sizes were markedly reduced in both groups receiving CoPP- pretreated hESC-CMs. In hearts receiving CoPPpretreated hESC-CMs alone, infarct size was limited to $32.4 \% \pm 2.5 \%$ of the ventricular circumference $(P \leq$ .02 vs each of the 2 groups without cell therapy). Adding a 4-week course of systemic CoPP to CoPP-pretreated hESC-CM injections produced a further decrease in the infarct area to $29.1 \% \pm 3.0 \%$ of the ventricle $(P<.0001$ vs the 2 groups without cell therapy and $P=.001$ vs hearts with PBS-pretreated hESC-CMs; Figure $3, D)$.

\section{Cell Therapy With CoPP-Pretreated hESC-CMs Preserves Infarct Wall Thickness}

Marked wall thinning was seen in infarcted hearts that received vehicle-only injections, reducing ventricular wall thickness to $43.1 \% \pm 4.0 \%$ of that in adjacent, uninfarcted heart wall (Figure 3,E). Cell therapy with PBS-pretreated hESC-CMs did not improve wall thickness at 8 weeks $(45.3 \% \pm 3.0 \%, P>.9999$ vs each of the 2 control groups without cell therapy). Also, a 4-week course of systemic CoPP alone (without cell therapy) did not improve wall thickness $(43.7 \% \pm 3.5 \%, P>.9999$ vs vehicle-only controls).

In contrast, wall thickness was much better preserved in hearts receiving CoPP-pretreated hESC-CM grafts, with or without systemic CoPP supplementation. Infarct 


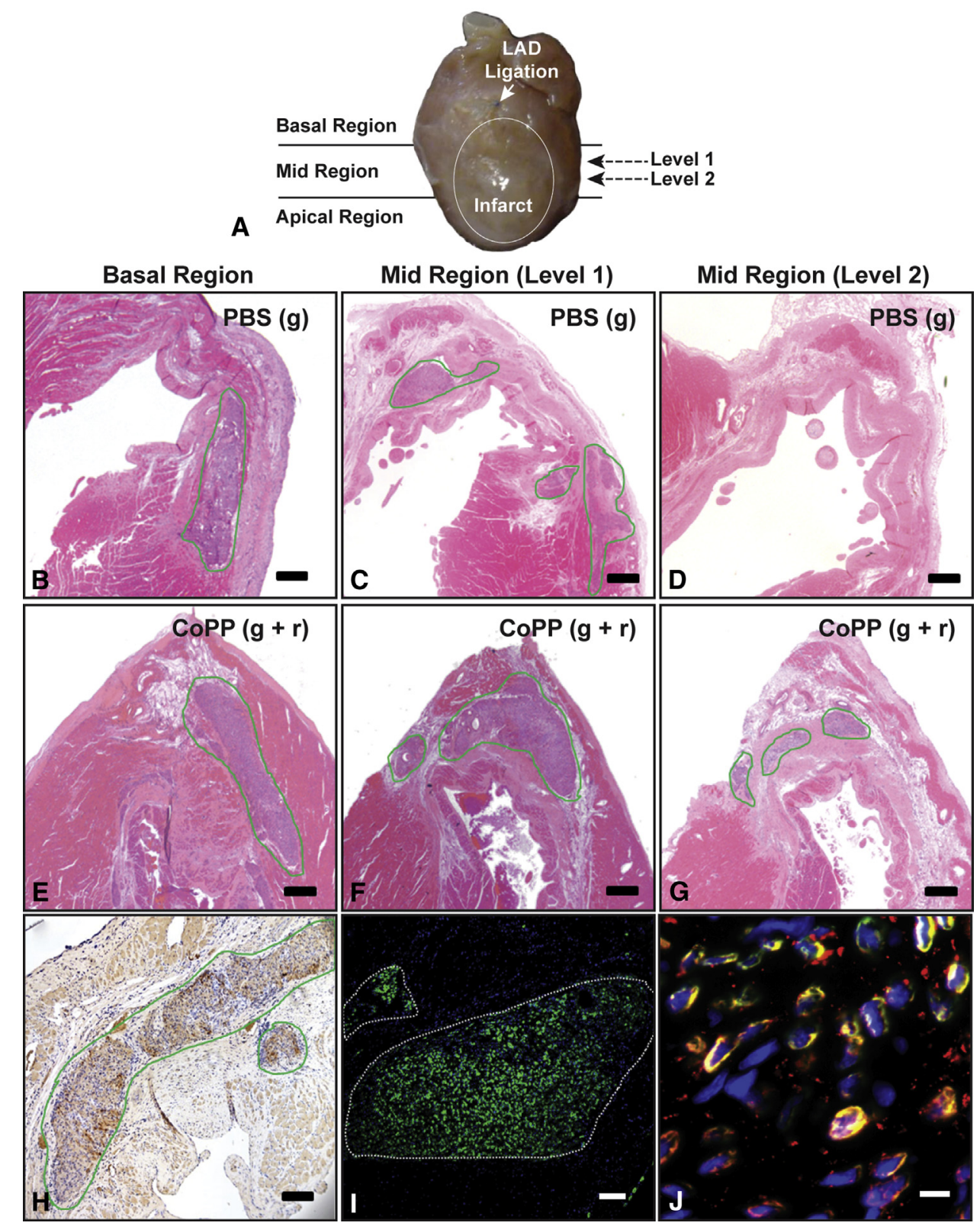

FIGURE 2. Histology of human embryonic stem cell-derived cardiomyocyte (hESC-CM) grafts at 8 weeks after transinfarct implantation. A, Sectioning diagram for fixed hearts to survey the infarct for the presence of human grafts along the diagonally oriented injection tract. Further serial sectioning was performed within each region to determine the extent of human cell engraftment in the infarct center (eg, levels 1 and 2). Hematoxylin and eosin staining of representative hearts from (B-D) the group that received phosphate-buffered saline (PBS)-pretreated hESC-CMs $[P B S(g)]$ and (E-G) the group that received cobalt protoporphyrin $(\mathrm{CoPP})$-pretreated hESC-CMs plus recipient treatment with systemic $\mathrm{CoPP}[\mathrm{CoPP}(g+r)]$. B and E, In basal regions, hESC-CM grafts in both treatment groups are seen extending into noninfarcted host myocardium. Note the lack of fibrotic encapsulation around the grafts. $\mathrm{C}$ and $\mathrm{F}$, In the mid-infarct region, grafts are found at the lateral infarct margins, near the infarct border zone, under both experimental conditions (level 1). However, grafts are present in the infarct center only in (F) the CoPP-treated group, where the potential for infarct replacement by graft and the creation of infarct-spanning bridges is evident. D and G, In deeper levels of the infarct (level 2), grafts are seen only in the CoPP-treated heart, with no graft present in the heart that received PBS-treated cells. Grafts were confirmed to be of human origin by immunostaining with $(\mathrm{H}) \beta$-myosin heavy chain (dark brown) and (I) human lamin A/C (green). J, Dual immunostaining demonstrating gap junction proteins (connexin 43, red) on graft cells (human lamin A/C, green), with yellow indicating color convergence. Scale bars $=500 \mu \mathrm{m}, 50 \mu \mathrm{m}, 25 \mu \mathrm{m}$, and $5 \mu \mathrm{m}$, for B-G, H, I, and J, respectively. $L A D$, Left anterior descending coronary artery. 

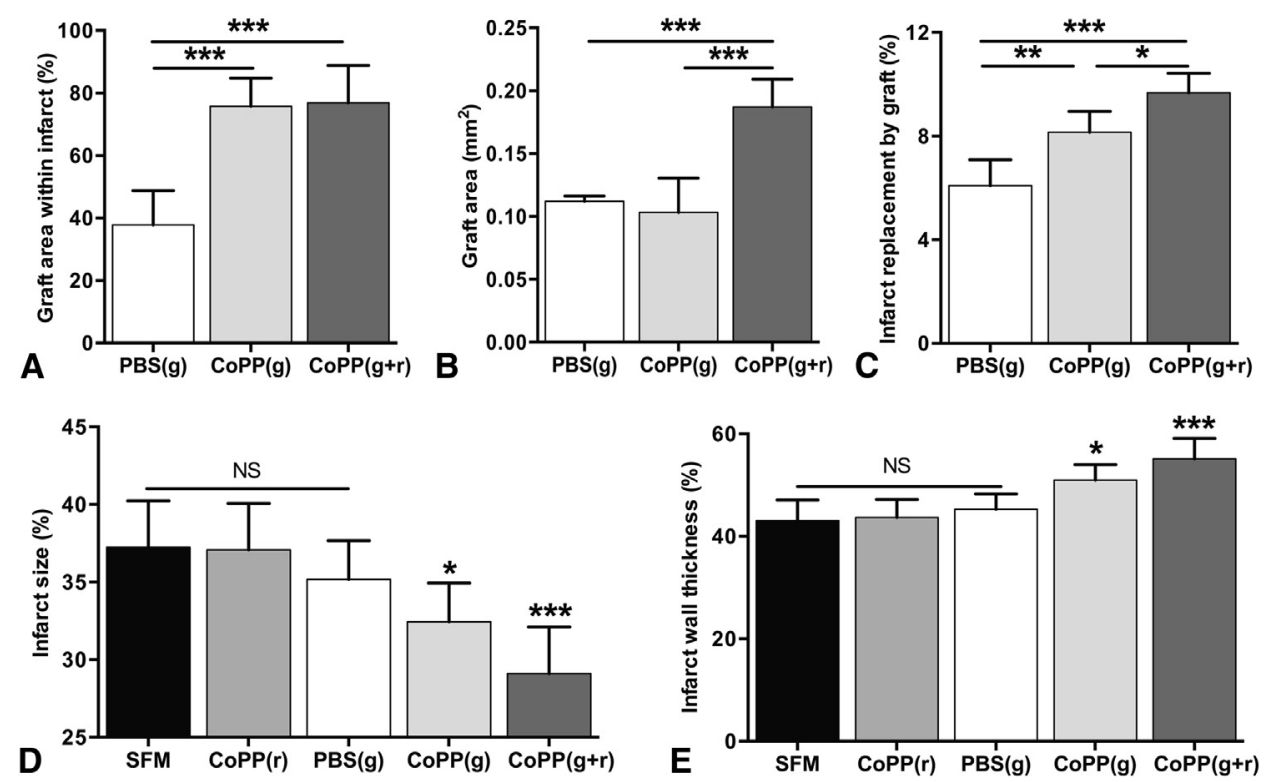

FIGURE 3. Left ventricular morphometry at 8 weeks after acute infarction and cell therapy. A, Percentage of the total graft area located within the infarct. $\mathrm{B}$, Mean graft areas cumulative over all surveyed sections/heart. C, Extent of total infarct area replaced by cellular graft. A-C, $* P<.01 ; * * P<.001$; $* * * P<.0001$ between indicated groups. D, Infarct size quantified as a percentage of the total left ventricular circumference. $* P<.02$ versus SFM and $\operatorname{CoPP}(\mathrm{r}) ; * * P \leq .0001$ versus SFM, CoPP(r), and PBS $(\mathrm{g})$. E, Mean wall thickness in the infarct area as a percentage of the wall thickness in the adjacent, noninfarcted left ventricle. $* P<.002$ versus SFM and $\operatorname{CoPP}(\mathrm{r})$; ***P $<.0001$ versus SFM, CoPP(r), and PBS(g). Data represent mean values \pm standard deviations; $\mathrm{n}=8$ /group. Treatment groups-postinfarct cell therapies: $P B S(g)$, Graft human embryonic stem cell-derived cardiomyocytes (hESC-CMs) pretreated with phosphate-buffered saline; $\operatorname{CoPP}(g)$, graft hESC-CMs pretreated with cobalt protoporphyrin $(\mathrm{CoPP})$; $C o P P(g+r)$, graft hESC-CMs pretreated with CoPP plus recipient treatment with systemic CoPP. Control groups without cell therapy: SFM, Infarct injections with serum-free media (vehicle-only control); $\operatorname{CoPP}(r)$, infarct recipients treated with systemic CoPP.

wall thickness was $51.0 \% \pm 3.0 \%$ of normal in hearts given CoPP-pretreated hESC-CMs $(P \leq .002$ vs the 2 groups without cell therapy, and $P=.03$ vs hearts with PBS-pretreated cells). In the group that received CoPP-pretreated hESC-CMs plus systemic CoPP, wall thickness was maintained at $55.1 \% \pm 4.0 \%$ of normal $(P<.0001$ vs the same 3 groups). Infarct areas in hearts that received CoPP-pretreated hESC-CMs plus systemic CoPP appeared well populated with cardiomyocytes, with resident cells consisting not only of graft cells but also of surviving native cardiomyocytes (Figure 2, F).

\section{Cell Therapy Improves Postinfarct Global Heart Function}

Global heart function, gauged by mean FS, decreased precipitously in all hearts within 48 hours after infarct creation (from a mean of $51.6 \% \pm 6.7 \%$ pre-MI to $25.5 \% \pm 4.9 \%$ at 48 hours post-MI; Figure $4, A$ ). In hearts receiving vehicle-only injections, the FS continued to decline by another $30 \%$ to $35 \%$ over the first month postinfarct, reaching $17.4 \% \pm 3.5 \%$ at 8 weeks $(P=.001$ at 4 weeks and $P=.015$ at 8 weeks vs 48 hours post-MI). In contrast, FS in all 3 groups of hearts receiving cell therapy at the time of infarction showed a $13 \%$ to $18 \%$ improvement by 4 and 8 weeks, with mean FS at 8 weeks increasing to $28.2 \% \pm 5.7 \%$. FS in each cell therapy group was notably higher than in the vehicle-only (SFM) group at both 4 weeks $(P \leq .0001)$ and 8 weeks $(P<.01$ for groups with PBS- and CoPP-pretreated grafts, $P<.001$ for the group receiving CoPP-pretreated hESC-CMs plus systemic CoPP). The protocol using CoPP combination therapy resulted in the highest FS at both 4 and 8 weeks $(31.0 \% \pm$ $6.4 \%$ and $29.3 \% \pm 5.0 \%$, respectively), although this was not statistically different from the 2 other cell therapy groups $(P>.0125)$.

The primary contributor to the improvement in ejection fraction was a reduction in end-systolic dimensions in hearts receiving cell therapy (Figure $4, B$ ). LVESDs in all treatment groups bettered vehicle-only controls at 4 weeks (each $P \leq .0002$ ). However, by 8 weeks, only the 2 groups with CoPP-pretreated cells had lower LVESDs than vehicle-injected groups (each $P \leq .002$ ). At 8 weeks, differences in LVEDDs between treatment groups were not statistically significant $(P>.0125)$, but, nonetheless, hearts in the 2 groups with CoPPpretreated cells also had the lowest LVEDDs (data not shown).

\section{Effects of Systemic CoPP Alone on Functional Parameters}

Weekly doses of CoPP, without cell therapy, also improved postinfarct ventricular function during ongoing 


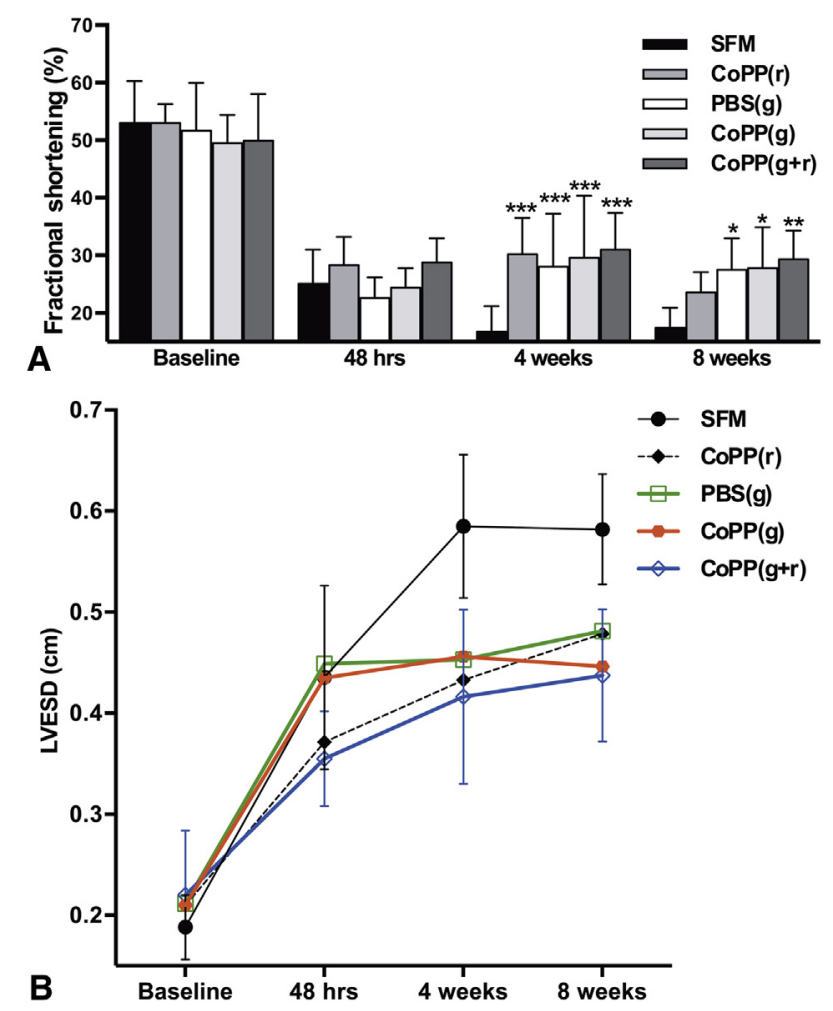

FIGURE 4. Echocardiographic assessment of functional parameters at baseline, 48 hours, and 4 and 8 weeks after infarction. A, Fractional shortening $(\%)$. Data represent mean values \pm standard deviations. ${ }^{*} P<.01 ;{ }^{* *} P \leq .001 ; * * * P \leq .0001$ versus SFM. B, LVESD. Points indicate mean values. Error bars showing standard deviations are limited to the 2 outermost groups so that trends in the mean values can be more easily visualized. $S F M$, Infarct injections with serum-free media (vehicle-only control); $L V E S D$, left ventricular end-systolic diameter; $C o P P(r)$, infarct recipients treated with systemic cobalt protoporphyrin (CoPP); $P B S(g)$, graft human embryonic stem cell-derived cardiomyocytes (hESC-CMs) pretreated with phosphate-buffered saline; $\operatorname{CoPP}(g)$, graft hESC-CMs pretreated with $\mathrm{CoPP} ; \operatorname{CoPP}(g+r)$, graft hESC-CMs pretreated with CoPP plus recipient treatment with systemic CoPP.

treatment. FS was higher and LVESD lower at 4 weeks in the group treated with systemic CoPP alone compared to vehicle-only controls (both $P \leq .0001$; Figure $4, A$ and $B$ ). Notably, at 4 weeks, rats receiving systemic CoPP had a FS of $30.2 \% \pm 6.3 \%$ compared to only $16.8 \% \pm 4.3 \%$ in controls given vehicle injections. However, the improvement in FS was a transient one, and lost at 8 weeks $(P>$ .0125), 1 month after the CoPP dosing had been discontinued.

\section{DISCUSSION}

Producing infarct-spanning bridges of neomyocardium, directionally aligned with the inherent helical myocardial bands of contraction, ${ }^{1}$ has the potential to correct postinfarct ventricular dysfunction in ways that are not currently possible. However, development of such techniques has been hampered by the early demise of cells implanted into infarcts. ${ }^{5-8}$ To assess the cytoprotective effects of CoPP pharmacotherapy, hESC-CMs were implanted under the most adverse conditions-immediately after acute infarct creation, directly into the infarct center, and into an infarct with permanent coronary occlusion (without reperfusion), conditions that have previously precluded hESC-CM survival. Thus, the most noteworthy consequence of CoPP pretreatment of hESC-CMs was that $76 \%$ of the resulting graft area at 8 weeks was found situated deep within the infarct center, double that of the PBS-treated cells, making the creation of infarct-spanning myocardial bridges feasible.

Some recipient groups were also given a course of systemic CoPP to extend the pharmacologic effects over the first month after implantation. Hearts receiving the combined therapy were found to have larger grafts and a greater extent of infarct replacement by grafted cells compared to the cell therapy groups without systemic CoPP, suggesting that continued expression of HO-1 (and perhaps nrf2-related antioxidants) ${ }^{14,15,21}$ may have further stabilized these nascent grafts during infarct maturation. Populations of surviving native myocytes were also seen within infarcts in hearts receiving combined therapy. This was not unexpected, given that induced HO-1 expression is known to improve native myocyte survival in the face of ischemia/infarction. ${ }^{16-18}$ In the present protocol, the injected CoPP-pretreated cells may have acted as delivery vehicles for HO-1 within the unvascularized infarct center, producing early local cytoprotective effects (eg, through released carbon monoxide) that would also have benefited resident host cardiomyocytes and endothelial cells.

Importantly, in addition to enhancing graft viability, injections of CoPP-pretreated hESC-CMs also positively affected ventricular remodeling in the native heart by reducing infarct size and LVESD while preserving wall thickness in the infarct region. Notably, these effects on remodeling were seen only in groups receiving CoPP-pretreated hESC-CMs and not in hearts receiving PBS-pretreated cells. Further, both improvements in infarct size and wall thickness were even more pronounced when cell therapy was accompanied by the concomitant course of systemic CoPP.

All hearts receiving cell therapy were found to have better ventricular function at 1 and 2 months, with or without CoPP treatment, compared to hearts with vehicle-only injections. Thus, the larger graft sizes and mitigated remodeling seen in hearts receiving CoPP-supplemented grafts did not translate into advantages in FS beyond those achieved by cell therapy without CoPP. However, direct mechanical contributions from these still-immature cardiomyocytes were not expected at this time. That said, trends revealed that, by 8 weeks, ventricular dimensions were stabilizing in the 2 groups 
receiving CoPP-pretreated cells while continuing to increase in hearts from other groups, suggesting that functional gains might occur over time as these larger intrainfarct grafts mature and proliferate, especially in models with graft-host conduction.

Given the known effects of HO-1 overexpression on postinfarct ventricular function and remodeling, ${ }^{16,19,34}$ systemic CoPP was given without cell therapy to an additional control group. In the absence of cell therapy, systemic CoPP did not alter the infarct size. However, infarcted hearts exposed to weekly systemic CoPP alone did exhibit markedly improved FS by 1 month. But, once systemic CoPP was discontinued, these early gains in global function were lost by 2 months. In contrast, improvements in heart function were sustained at 2 months only when the peri-infarction course of systemic CoPP was combined with cell therapy.

Limitations of the present study are the xenograft model (without graft-host conduction) and caveats regarding extrapolation of rodent data to humans. However, a new report on infarct repair in nonhuman primate hearts has demonstrated hESC-CM graft survival at 3 months with electrical coupling to host cardiomyocytes, ${ }^{35}$ raising expectations that cellular transplants can persist and integrate into host ventricles. If so, such a clinically relevant model could be used to determine whether structural purposing of cellular grafts by the proposed strategies would offer long-term functional advantages. As CoPP alternatives, other less specific, but Food and Drug Administration-approved, HO-1 inducers (eg, statins, phosphodiesterase-5 inhibitors) might be explored. In future clinical applications, such infarct-spanning "myobridges" could be surgically implanted during coronary artery bypass grafting or minimally invasive interventions for heart failure. Preprocedural imaging and predictive simulation modeling might even allow band orientation to be planned and optimized for individual hearts.

The authors thank Benjamin van Biber for stem cell preparation, Drs Robert Welikson and Virginia M. Green for reviewing and editing our manuscript; Dr Jason M. Kim for assistance with surgical procedures; and Dr Pamela Johnson and Mary Beauchamp for preparation of the tissue sections.

\section{References}

1. Torrent-Guasp F, Ballester M, Buckberg GD, Carreras F, Flotats A, Carrio I, et al. Spatial orientation of the ventricular muscle band: physiologic contribution and surgical implications. J Thorac Cardiovasc Surg. 2001;122:389-92.

2. Buckberg G, Mahajan A, Saleh S, Hoffman JI, Coghlan C. Structure and function relationships of the helical ventricular myocardial band. J Thorac Cardiovasc Surg. 2008;136:578-89, 589.e1-11.

3. Buckberg GD. Basic science review: the helix and the heart. J Thorac Cardiovasc Surg. 2002; 124:863-83.

4. Spotnitz HM. Macro design, structure, and mechanics of the left ventricle. J Thorac Cardiovasc Surg. 2000;119:1053-77.

5. Zhang M, Methot D, Poppa V, Fujio Y, Walsh K, Murry CE. Cardiomyocyte grafting for cardiac repair: graft cell death and anti-death strategies. $J$ Mol Cell Cardiol. 2001;33:907-21.
6. Robey TE, Saiget MK, Reinecke H, Murry CE. Systems approaches to preventing transplanted cell death in cardiac repair. J Mol Cell Cardiol. 2008;45:567-81.

7. Chimenti I, Smith RR, Li TS, Gerstenblith G, Messina E, Giacomello A, et al. Relative roles of direct regeneration versus paracrine effects of human cardiosphere-derived cells transplanted into infarcted mice. Circ Res. 2010; 106:971-80.

8. Terrovitis JV, Smith RR, Marban E. Assessment and optimization of cell engraftment after transplantation into the heart. Circ Res. 2010;106:479-94.

9. Li RK, Mickle DA, Weisel RD, Rao V, Jia ZQ. Optimal time for cardiomyocyte transplantation to maximize myocardial function after left ventricular injury. Ann Thorac Surg. 2001;72:1957-63.

10. Laflamme MA, Chen KY, Naumova AV, Muskheli V, Fugate JA, Dupras SK et al. Cardiomyocytes derived from human embryonic stem cells in prosurvival factors enhance function of infarcted rat hearts. Nat Biotechnol. 2007; 25:1015-24.

11. Lee ST, White AJ, Matsushita S, Malliaras K, Steenbergen C, Zhang Y, et al. Intramyocardial injection of autologous cardiospheres or cardiosphere-derived cells preserves function and minimizes adverse ventricular remodeling in pigs with heart failure post-myocardial infarction. J Am Coll Cardiol. 2011;57: 455-65.

12. Luo J, Weaver MS, Cao B, Dennis JE, Van Biber B, Laflamme MA, et al. Cobal protoporphyrin pretreatment protects human embryonic stem cell-derived cardiomyocytes from hypoxia/reoxygenation injury in vitro and increases graft size and vascularization in vivo. Stem Cells Trans Med. 2014;3:734-44.

13. Kawamoto S, Flynn JP, Shi Q, Sakr SW, Luo J, Allen MD. Heme oxygenase-1 induction enhances cell survival and restores contractility to unvascularized three-dimensional adult cardiomyocyte grafts implanted in vivo. Tissue Eng Part A. 2011;17:1605-14

14. Piantadosi CA, Carraway MS, Babiker A, Suliman HB. Heme oxygenase-1 regulates cardiac mitochondrial biogenesis via Nrf2-mediated transcriptional control of nuclear respiratory factor-1. Circ Res. 2008;103:1232-40.

15. Cai C, Teng L, Vu D, He JQ, Guo Y, Li Q, et al. The heme oxygenase 1 induce (CoPP) protects human cardiac stem cells against apoptosis through activation of the extracellular signal-regulated kinase (ERK)/NRF2 signaling pathway and cytokine release. J Biol Chem. 2012;287:33720-32.

16. Melo LG, Agrawal R, Zhang L, Rezvani M, Mangi AA, Ehsan A, et al. Gene therapy strategy for long-term myocardial protection using adeno-associated virus-mediated delivery of heme oxygenase gene. Circulation. 2002;105:602-7.

17. Tang YL, Qian K, Zhang YC, Shen L, Phillips MI. A vigilant, hypoxia-regulated heme oxygenase-1 gene vector in the heart limits cardiac injury after ischemiareperfusion in vivo. J Cardiovasc Pharmacol Ther. 2005;10:251-63.

18. Yet SF, Tian R, Layne MD, Wang ZY, Maemura K, Solovyeva M, et al. Cardiacspecific expression of heme oxygenase- 1 protects against ischemia and reperfusion injury in transgenic mice. Circ Res. 2001;89:168-73.

19. Liu X, Simpson JA, Brunt KR, Ward CA, Hall SR, Kinobe RT, et al. Preemptive heme oxygenase-1 gene delivery reveals reduced mortality and preservation of left ventricular function 1 year after acute myocardial infarction. Am J Physiol Heart Circ Physiol. 2007;293:H48-59.

20. Abraham NG, Kappas A. Pharmacological and clinical aspects of heme oxygenase. Pharmacol Rev. 2008;60:79-127.

21. Gozzelino R, Jeney V, Soares MP. Mechanisms of cell protection by heme oxygenase-1. Annu Rev Pharmacol Toxicol. 2010;50:323-54.

22. Thomson JA, Itskovitz-Eldor J, Shapiro SS, Waknitz MA, Swiergiel JJ, Marshall VS, et al. Embryonic stem cell lines derived from human blastocysts Science. 1998;282:1145-7.

23. Chachques JC, Cattadori B, Herreros J, Prosper F, Trainini JC, Blanchard D, et al. Treatment of heart failure with autologous skeletal myoblasts. Herz. 2002;27:570-8

24. Akamatsu Y, Haga M, Tyagi S, Yamashita K, Graca-Souza AV, Ollinger R, et al. Heme oxygenase-1-derived carbon monoxide protects hearts from transplant associated ischemia reperfusion injury. FASEB J. 2004;18:771-2.

25. Lakkisto P, Kyto V, Forsten H, Siren JM, Segersvard H, Voipio-Pulkki LM, et al Heme oxygenase- 1 and carbon monoxide promote neovascularization after myocardial infarction by modulating the expression of HIF-1alpha, SDF1alpha and VEGF-B. Eur J Pharmacol. 2010;635:156-64.

26. Katori M, Buelow R, Ke B, Ma J, Coito AJ, Iyer S, et al. Heme oxygenase-1 overexpression protects rat hearts from cold ischemia/reperfusion injury via an antiapoptotic pathway. Transplantation. 2002;73:287-92.

27. L'Abbate A, Neglia D, Vecoli C, Novelli M, Ottaviano V, Baldi S, et al. Beneficial effect of heme oxygenase-1 expression on myocardial ischemia-reperfusion involves an increase in adiponectin in mildly diabetic rats. Am J Physiol Heart Circ Physiol. 2007;293:H3532-41. 
28. Negroni E, Riederer I, Chaouch S, Belicchi M, Razini P, Di Santo J, et al. In vivo myogenic potential of human CD133(+) muscle-derived stem cells: a quantitative study. Mol Ther. 2009;17:1771-8.

29. Pfeffer MA, Pfeffer JM, Fishbein MC, Fletcher PJ, Spadaro J, Kloner RA, et al. Myocardial infarct size and ventricular function in rats. Circ Res. 1979;44: 503-12.

30. Takagawa J, Zhang Y, Wong ML, Sievers RE, Kapasi NK, Wang Y, et al Myocardial infarct size measurement in the mouse chronic infarction model comparison of area- and length-based approaches. J Appl Physiol (1985) 2007; 102:2104-11.

31. Fishbein MC, Maclean D, Maroko PR. Experimental myocardial infarction in the rat: qualitative and quantitative changes during pathologic evolution. Am J Pathol. 1978:90:57-70.
32. Dai W, Wold LE, Dow JS, Kloner RA. Thickening of the infarcted wall by collagen injection improves left ventricular function in rats: a novel approach to preserve cardiac function after myocardial infarction. J Am Coll Cardiol. 2005;46:714-9.

33. Laird NM, Ware JH. Random-effects models for longitudinal data. Biometrics. 1982;38:963-74.

34. Lakkisto P, Siren JM, Kyto V, Forsten H, Laine M, Pulkki K, et al. Heme oxygenase-1 induction protects the heart and modulates cellular and extracellular remodelling after myocardial infarction in rats. Exp Biol Med (Maywood). 2011; 236:1437-48.

35. Chong JJ, Yang X, Don CW, Minami E, Liu YW, Weyers JJ, et al. Human embryonic-stem-cell-derived cardiomyocytes regenerate non-human primate hearts. Nature. 2014;510:273-7.

\title{
EDITORIAL COMMENTARY
}

\section{Cardiac stem cell therapy: Checkered past, promising future?}

\author{
John J. Squiers, BSE, ${ }^{\mathrm{a}}$ Kelley A. Hutcheson, MD, ${ }^{\mathrm{b}}$ Jeffrey E. Thatcher, $\mathrm{PhD},{ }^{\mathrm{c}}$ and J. Michael DiMaio, MD ${ }^{\mathrm{c}, \mathrm{d}}$
}

See related article on pages 3180-8.

In this issue of the Journal of Thoracic and Cardiovascular Surgery (JTCVS), Luo and colleagues ${ }^{1}$ have demonstrated a novel approach to transplantation of human embryonic stem cell grafts within infarcted myocardium in an animal model. We commend their emphasis on transplanting stem cell grafts as directionally oriented, infarct-spanning bands. Often missing from basic science laboratories is the understanding of the importance of these macroscopic helical Torrent-Guasp myocardial bands ${ }^{2}$ that allow for torsional deformation of the left ventricle during systole and, thus, appropriate ventricular function. By submitting their work to JTCVS, Luo and colleagues recognize that cardiac surgeons, who handle the human heart on a near daily basis, can best appreciate the importance of the heart's macroarchitecture for effective cardiac function.

Their demonstrated effects of CoPP preconditioning represent an important step forward in our understanding of the basic mechanisms and principles underlying regenerative stem cell therapy. This bench-top research is crucial to

From the University of Texas Southwestern Medical Center, ${ }^{a}$ Dallas, Tex; The Heart Hospital of Baylor, ${ }^{\mathrm{b}}$ Plano, Tex; Spectral MD ${ }^{\mathrm{c}}$ Dallas, Tex; and Baylor University Medical Center, ${ }^{\mathrm{d}}$ Dallas, Tex.

The authors thank Brian J. Baldwin, MD for his helpful advice and feedback on the paper, Kristin Yang for her illustrations in Figures 1 and 2, and Leah Gaither for editorial assistance.

Disclosures: Authors have nothing to disclose with regard to commercial support.

Received for publication Oct 16, 2014; accepted for publication Oct 17, 2014

Address for reprints: J. Michael DiMaio, MD, Baylor University Medical Center, 6125 Luther Lane, Dallas, TX 75225 (E-mail: jmdimaio@yahoo.com).

J Thorac Cardiovasc Surg 2014;148:3188-93

$0022-5223 / \$ 36.00$

Copyright (c) 2014 by The American Association for Thoracic Surgery

http://dx.doi.org/10.1016/j.jtcvs.2014.10.077 assist clinicians in achieving better clinical outcomes with cardiac stem cell therapy. In light of this success, however, it is essential to recognize the often-frustrating discordance between results of promising basic science research and more humbling clinical trials over the last 15 years of investigation into stem cell therapy for cardiac disease.

Extensive research efforts have investigated the potential for stem cell-mediated cardiac tissue regeneration in the treatment of cardiovascular disease. ${ }^{3}$ Early basic science reports suggested enormous therapeutic potential, ${ }^{4}$ but results from clinical trials have been disappointing to date. Basic investigators have employed various kinds of stem cells: embryonic stem cells (ESCs), induced pluripotent stem cells (iPSCs), and adult progenitor cells (including skeletal myoblasts, bone marrow mononuclear cells [BMMNCs], and cardiac stem cells [CSCs]). So far, however, completed clinical trials have only reported transplantation of the various adult progenitor cells. In fact, a casual review of the clinical trials database (clinicaltrials.gov/ct2/home) shows that the overwhelming majority of trials has employed or will employ adult progenitor stem cells rather than truly pluripotent ESCs or iPSCs.

An early clinical trial of skeletal myoblasts (Myoblast Autologous Grafting in Ischemic Cardiomyopathy [MAGIC $^{5}$ ) was discontinued prematurely after both a lack of efficacy and an increased risk for arrhythmias in patients receiving stem cell therapy were demonstrated. A more recent skeletal myoblast trial (ie, SEISMIC ${ }^{6}$ ) did not reveal any effect of stem cell therapy on the leftventricular ejection fraction (LVEF). Although BMMNCs have been the most commonly employed cell type for stem cell therapy trials (eg, REPAIR-AMI, ${ }^{7}$ TIME, ${ }^{8}$ POSEIDON $^{9}$ ), these investigations have also yielded underwhelming results. Meta-analyses of BMMNC trials 

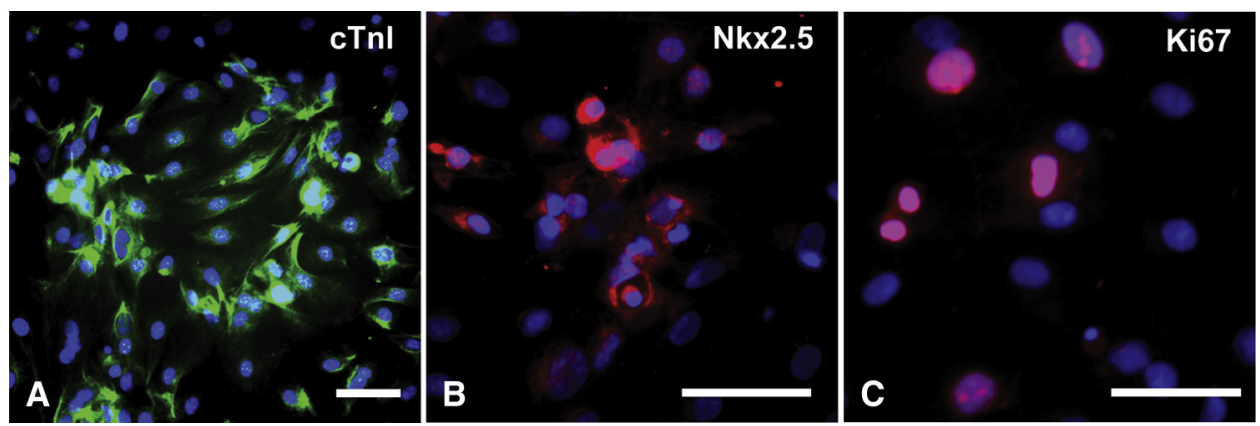

FIGURE E1. Characterization of cell injectates prior to implantation. Aliquots of differentiated human embryonic stem cell-derived cardiomyocyte (hESC-CM) injectates were stained with antibodies to (A) cardiac troponin I, denoting cardiomyocytes (green fluorescence); B, Nkx 2.5 , an early cardiomyocyte-specific transcription factor (red); or C, Ki67, indicating mitotic cells (red). The nuclei in all panels were counterstained with Hoechst dye (blue). Scale bars $=50 \mu \mathrm{m}$. 